\section{Der Streit der Gutachter}

\section{Die Vorschläge zur Neuordnung der europäischen Chemikalienpolitik haben heftige Reaktionen ausgelöst. Geprägt wird die Diskussion von der erwarteten Kostenbelastung. Eine ausgewogene Betrachtung darf die möglichen Nutzen- effekte gleichwohl nicht vernachlässigen.}

$\mathbf{I}$ Von Ralf Nordbeck und Torsten Frobwein m Mai 2003 hat die Europäische Kommission einen Entwurf für die zukünftige EUChemikalienregulierung zur Konsultation vorgelegt. Zentrales Element bildet ein neues System für die Chemikalienkontrolle namens REACH. Das REACH-System setzt sich aus den Komponenten Registrierung, Bewertung und Zulassung zusammen, die gegenüber der bisherigen Regelung einen Mehraufwand für Unternehmen bedeuten, aber auch erhebliche positive Umweltauswirkungen haben (1). In den Monaten vor der Veröffentlichung des Verordnungsentwurf sind die Reaktionen auf die zukünftige europäische Chemikalienpolitik in zunehmenden Maße kontrovers verlaufen.

Im Rahmen der achtwöchigen Konsultation sind etwa 6.400 Beiträge eingegangen. Fast alle Stellungnahmen von Unternehmen, Verbänden und Gewerkschaften äußern sich sehr kritisch zum Verordnungsentwurf. Befürchtet werden negative wirtschaftliche Auswirkungen, der Verlust von Arbeitsplätzen und eine Beeinträchtigung der internationalen Wettbewerbsfähigkeit. Die im Entwurf vorgeschlagenen administrativen Verfahren und die finanziellen Belastungen werden gerade für kleine und mittlere Unternehmen als nicht tragbar bewertet.

Die europäischen Umwelt- und Verbraucherschutzverbände sowie deutsche Gewerkschaften werfen der Industrie hingegen gezielte Desinformation und Schwarzmalerei vor. Die Schätzungen der Kosten für die neue Chemikalienregulierung seien systematisch überhöht, während die Nutzeneffekte der neuen Chemikalienpolitik keine Berïcksichtigung fänden.

\section{- Nułzenschätzungen}

Als Ziel der Reform der europäischen Chemikalienregulierung nennt die Kommission eine Verbesserung des Schutzniveaus von menschlicher Gesundheit und der Umwelt. Aktuelle Studien geben einen ersten Aufschluss darüber, inwieweit sich mögliche Nutzenwirkungen der neuen Chemikalienregulierung in vermiedenen Kosten für Gesundheits- und Umweltschäden, Produktionsausfällen und Haftungsansprïchen ausdrücken lassen. Hinzuzurechnen sind zukünftig zu erwartende Nutzen durch nachhaltige Innovationen der Chemieindustrie.

Pearce/Koundouri ermitteln die gesellschaftlichen Kosten von Krankheitsfällen und einer verminderten Lebenserwartung, die durch eine effektive Chemikalienkontrolle vermieden werden könnten (1). Eine erweiterte Analyse berïcksichtigt die durch Krankheit und verkürzte Lebenserwartung bedingten Produktionsausfälle und Produktivitätsverluste. Insgesamt ermitteln die Autoren eine Bandbreite vermeidbarer Kosten bis zum Jahr 2020 in Größenordnungen von 4,8 bis 283,5 Milliarden Euro. Auch die von der Kommission angegebenen jährlichen Gesundheitskosten in Höhe von 29 Milliarden Euro für die Behandlung von Allergien können zum Teil gesenkt werden. Neben den gesellschaftlichen Einsparungen sind auch direkte Kostenvermeidungen für Unternehmen darstellbar. In einer kürzlich veröffentlichten Studie beziffern Risk \& Policy Analysts (RPA) mögliche Kosteneinsparungen im gesundheitsbezogenen Arbeitsschutz in Höhe von 17,6 bis 54,4 Milliarden Euro über eine Zeitspanne von 30 Jahren (3). Der für Unternehmen wirksame ökonomische Nutzen wurde aus der Vermeidung von Kosten für medizinische Behandlungen und von Produktionsausfällen sowie individueller Zahlungsbereitschaften zur Vermeidung von Gesundheitsschäden prognostiziert. Eine frühere Studie im Auftrag des britischen Umweltministeriums gelangt auf Einsparungen durch Vermeidung von Unfällen und der Verringerung des Risikos für berufsbedingte Asthma- und Dermatitiserkrankungen von bis zu 1,3 Milliarden britischen Pfund. Studien mit belastbaren Ergebnissen zu vermeidbaren Schäden für die Umwelt sind aufgrund methodischer Probleme und einer Vielzahl wechselseitiger Einflussfaktoren kaum erhältlich. Trotz dieser Einschränkungen leiten RPA allein bei der Anwendung der REACH-Systematik auf vier umweltgefährdende Stoffe (Tributylzinn, kurzkettige Chlorparaffine, Tetrachlorethylen, Nonylphenol) eine Vielzahl an vermeidbaren Umweltschäden ab (4). Die diskutierten Umweltschäden sind nicht monetarisiert worden.

Ebenso unbeziffert bleiben Nutzenwirkungen für Unternehmen der Chemieindustrie aus den geänderten Rahmenbedingungen für Innovationen. Der Rat von Sachverständigen für Umweltfragen weist auf positive Innovationswirkungen hin, die sich an der neu geschaffenen Gleichbehandlung von Neu- und Altstoffen, der Anhebung der Volumenschwelle für die Registrierung von Neustoffen und der möglichen internationalen Vorreiterrolle des EU-Binnenmarktes für risikofreiere Stoffe orientieren (5).

\section{Kostenschätzungen}

Die neue Chemikalienregulierung belastet die betroffenen Unternehmen mit direkten und indirekten Kosten. Direkte Kosten entstehen für Hersteller und Importeure chemischer Stoffe aus der Bindung an das Registrierungs- und Zulassungsverfahren des REACH-Systems. Bei der Veröffentlichung des Weißbuches zur Reform der europäischen Chemikalienpolitik ging die Kommission zunächst von direkten Kosten in Höhe von 2,1 Milliarden Euro innerhalb von 10 Jahren aus. In einer detaillierteren Analyse der Reformvorschläge kommt eine Studie von RPA zu direkten Kosten des REACH-Systems, die je nach Ausgestaltung der zukünftigen Verordnung 1,4 bis 7,8 Milliarden Euro betragen (6). Ein Wert von 4,1 Milliarden Euro wird als am wahrscheinlichsten erachtet. In einer weiteren Studie für das britische Umweltministerium beziffert RPA die direkten Kosten für die britische Wirtschaft auf 620 Millionen Pfund (7). Der europäische Verband der Chemischen Industrie kommt demgegenüber in seiner Schätzung bei $30.000 \mathrm{zu}$ testenden Stoffen auf direkte Kosten in Höhe von 7 bis 10 Milliarden Euro, die sich bei 70.000 Stoffen auf eine Größenordnung von 15 bis 20 Milliarden Euro erhöhen sollen (8).

Zusätzlich entstehen durch REACH indirekte Kosten bei den nachgeschalteten Anwendern. Diese indirekten Kosten ergeben sich aus höheren Chemikalienpreisen durch die Überwälzung der Prüfund Registrierungskosten auf die Anwender und den Folgen von Produktrationalisierungen und 
Stoffbeschränkungen, die die Notwendigkeit der Substitution nicht mehr verfügbarer Stoffe nach sich zieht. Die Kommission hat die indirekten Kosten für das verarbeitende Gewerbe sowie industrielle und private Anwendern bis 2020 auf 14 bis 26 Milliarden Euro veranschlagt (9).

Eine Studie zu indirekten Kosten und gesamtwirtschaftlichen Auswirkungen von REACH für die deutsche Wirtschaft hat Arthur D. Little im Auftrag des BDI vorgelegt (10). In verschiedenen Szenarien erwartet die Studie wirtschaftliche Beeinträchtigungen, bis hin zu extrem hohen gesamtgesellschaftlichen Schäden. Ausgehend von Produktionsverlusten des verarbeitenden Gewerbes von 1,4 bis 20,2 Prozent halten die Autoren der Studie einen Bruttowertschöpfungsverlust von 0,4 bis 6,4 Prozent für wahrscheinlich. Durch die übermäßige Belastung gerade kleiner und mittelgroßer Unternehmen werden Arbeitsplatzverluste von 150.000 bis 2,35 Millionen für die Gesamtwirtschaft geschlussfolgert.

Eine Studie der Unternehmensberatung Mercer Consulting kommt für Frankreich zu einer ähnlich negativen Einschätzung der wirtschaftlichen Auswirkungen des REACH-Systems (11). Auch diese Studie betont eine besondere Belastung kleinvolumiger Stoffe im Bereich der Fein- und Spezialchemie sowie kleiner und mittelgroßer Unternehmen. In Abhängigkeit der getroffenen Annahmen über die Ausgestaltung des Verordnungstextes rechnet Mercer für die heimische chemische Industrie mit einem Rückgang der Wertschöpfung, gemessen am Umsatz, von bis zu 11,5 Prozent. Innerhalb von zehn Jahren erachtet Mercer einen Rückgang der gesamtwirtschaftlichen Bruttowertschöpfung von 1,7 bis 3,9 Prozent und Arbeitsplatzverluste von über 800.000 Stellen als möglich.

In einer neuen Folgenabschätzung korrigiert die Kommission ihre früheren Schätzungen zu den Gesamtkosten der neuen Regulierung deutlich nach unten (12). Verteilt auf elf Jahre schätzt die Kommission die Gesamtkosten für die chemische Industrie und die nachgeschalteten Anwender nun auf 2,8 bis 5,2 Milliarden Euro. In diese Schätzungen eingeschlossen sind die direkten Kosten für die chemische Industrie in Höhe von 2,3 Milliarden Euro.

\section{- Kontroverse Gesamtbewertung}

Die vorliegenden Schätzungen zu den Kosten und Nutzen der neuen Chemikalienregulierung weisen erhebliche Unsicherheiten auf. Dies ist zum einen bedingt durch methodische Schwierigkeiten bei der Erfassung und Abschätzung aller relevanten Kosten- und Nutzenkategorien, zum anderen aber durch zum Teil willkürlich gesetzte Annahmen und eklatante methodische Schwächen in einigen Studien.

\section{- Quantifizierung von Effekten schwierig}

Die große Bandbreite bei den Nutzenschätzungen verdeutlicht, wie schwierig sich die Quantifizierung der Nutzeneffekte der neuen Chemikalienregulierung für die Umwelt und menschliche Gesundheit gestaltet. Gerade die Studie von RPA für das britische Umweltministerium zeigt jedoch, dass der Nutzen von 1,3 Milliarden Pfund die Kosten von 620 Millionen Pfund deutlich übersteigen kann. Auch die Studie von Pearce und Koundouri kommt insgesamt zu dem vorsichtigen Schluss, dass der Nutzen des REACH-Systems langfristig die Kosten übersteigen wird. Überdies geben die vorliegenden Schätzungen einen Ausblick auf das tatsächliche Ausmaß der möglichen Schäden, wird berücksichtigt, dass sie nicht auf alle Nutzenaspekte Bezug nehmen bzw. sich nur auf eine geringe Zahl ausgewählter Stoffe beschränken.

Die bisherigen Kostenschätzungen erscheinen insgesamt als zu hoch angesetzt. Einsparpotentiale durch bereits vorhandene Daten, alternative Testmethoden und Lerneffekte im Zeitverlauf werden in den vorliegenden Studien nicht ausreichend berücksichtigt. Die in den Studien von ADL und Mercer prognostizierten gesamtwirtschaftlichen Auswirkungen erscheinen zudem wenig plausibel. Die aktuelle Folgenabschätzung der Kommission zeigt überdies, dass die reduzierten Prüfanforderungen und vereinfachten Registrierungsverfahren sowie Erleichterungen für die nachgeschalteten Anwender im neuen Verordnungsentwurf in deutlich niedrigeren Kostenbelastungen resultieren.

\section{Der Autor}

Ralf Nordbeck und Thorsten Frohwein sind wissenschaftliche Mitarbeiter am Umweltforschungszentrum Leipzig-Halle (UFZ).

Kontakt: UFZ, Postfach 500136, 04301 Leipzig.

Tel.341-2352895, E-Mail: nordbeck@alok.ufz.de

\section{Anmerkungen}

(1) Für eine Erläuterung des REACH-Verfahrens siehe: Ahrens, A.: Die Kräfte des Marktes nutzbar machen. In: Ökologisches Wirtschaften, 5/2002.

(2) Pearce/Koundouri: The Social Cost of Chemicals. London 2003

(3) RPA: Assessment of the Impact of the New Chemicals Policy on Occupational Health. Loddon 2003.

(4) RPA: The Impact of the New Chemicals Policy on Health and the Environment. Loddon 2003.

(5) SRU: Zur Wirtschaftsverträglichkeit der Reform der Europäischen Chemikalienpolitik. Berlin 2003.

(6) RPA: Combined Costs of REACH and Entity Fees. Loddon 2003.

(7) RPA: Regulatory Impact Assessment of the EU White Paper. Loddon 2001.

(8) CEFIC: Barometer of Competitiveness - White Paper on Chemicals Policy. 2001.

(9) EU-Kommission: Fragen und Antworten zur neuen Chemikalienpolitik. Brüssel 2003

(10) ADL: Economic Effects of the EU Substances Policy. Wiesbaden 2003.

(11) Mercer Consulting: Study of the Impact of the Future Chemicals Policy. 2003

(12) EU-Kommission: Folgenabschätzung zur Chemikalienregulierung. Brüssel 2003.

\section{Anzeige}


(c) 20I0 Authors; licensee IÖW and oekom verlag. This is an article distributed under the terms of the Creative Commons Attribution Non-Commercial No Derivates License (http://creativecommons.org/licenses/by-nc-nd/3.o/), which permits unrestricted use, distribution, and reproduction in any medium, provided the original work is properly cited. 